\title{
Effective mass of two-dimensional electron gas in an $\mathrm{Al}_{0.2} \mathrm{Ga}_{0.8} \mathrm{~N} / \mathrm{GaN}$ heterojunction
}

\author{
D. R. Hang, C.-T. Liang, C. F. Huang, Y. H. Chang, and Y. F. Chen \\ Department of Physics, National Taiwan University, Taipei, Taiwan, 106 Republic of China \\ H. X. Jiang and J. Y. Lin \\ Department of Physics, Kansas State University, Manhattan, Kansas 66505-2601
}

(Received 29 September 2000; accepted for publication 27 April 2001)

\begin{abstract}
We have performed a magnetotransport study on an $\mathrm{AlGaN} / \mathrm{GaN}$ heterostructure at low temperatures. The effective-mass values have been evaluated by analyzing the exact form of the temperature-dependent Shubnikov-de Haas oscillation function. The values obtained increase with the magnetic field. This mass enhancement is attributed to conduction-band nonparabolicity. The effective-mass variation with the magnetic field was extrapolated to zero field, together with further correction due to the triangular confinement of the carriers, yielding an effective mass of 0.185 \pm 0.005 of the free-electron mass. Our result is in excellent agreement with the results obtained by first-principle calculations and the tight-binding method, and suggest the significance of magnetic-field-induced nonparabolicity in transport measurements. (c) 2001 American Institute of Physics. [DOI: 10.1063/1.1380245]
\end{abstract}

Recently, there have been increasing experimental studies aimed at clarifying the properties of group-III nitrides. These materials have the advantages of band structures of a direct-transition nature and widely varied band gaps. They are suitable for fabricating blue and ultraviolet light-emitting and laser diodes. Furthermore, their structures will be stronger and more chemically stable, and the devices made up of them can be operated in a high-power and in hightemperature environment. One of the important systems is the $\mathrm{AlGaN} / \mathrm{GaN}$ two-dimensional electron gas (2DEG) for its promising future in high electron mobility transistors.

From the technological point of view, some important electron parameters must be clarified, such as the electron effective mass, which is of crucial importance to optimize device performance. However, widely scattered values of the effective mass have been reported in previously published reports. ${ }^{1-5}$ The reported experimental results were somewhat larger than the predictions of theoretical calculations. ${ }^{6,7}$ The authors compensated the discrepancies between theory and experiment by including the nonparabolicity resulting from different carrier densities and spatial confinements. Yet, the improvements are not quite satisfactory. Recently, the reported values have ranged from 0.215 to $0.23 m_{0} \cdot{ }^{3,4}$ The purpose of this study is to elucidate the nature of the discrepancies between the experimental outcomes and theoretical calculations. We performed temperature-dependent Shubnikov-de Haas $(\mathrm{SdH})$ measurements to determine the electron effective mass in the interface. Contrary to previous studies, which assumed that the effective mass is a constant and independent of the magnetic field, we studied the variation of the effective mass with the magnetic field. We obtained the nonlinear variation of the effective mass and attributed it to the conduction-band nonparabolicity. Thus, we demonstrate the significance of the nonparabolicity effect for two-dimensional (2D) electrons confined at the $\mathrm{AlGaN} / \mathrm{GaN}$ heterojunction.

The sample used in this study was grown by metalorganic chemical-vapor deposition. A GaN buffer of $25 \mathrm{~nm}$ thickness was grown on the sapphire substrate, and it was followed by a $1.3-\mu$ m-thick $\mathrm{GaN}$, a 5 -nm-thick $\mathrm{Al}_{0.2} \mathrm{Ga}_{0.8} \mathrm{~N}$ barrier layer, and finally, by 25-nm-thick Si-doped $\mathrm{Al}_{0.2} \mathrm{Ga}_{0.8} \mathrm{~N}$. The one-side doping results in the triangular confinement of carriers in the heterojunction. The mobility is $1900 \mathrm{~cm}^{2} / \mathrm{V} \mathrm{s}$ at $77 \mathrm{~K}$. The properties of the 2DEG at the $\mathrm{Al}_{0.2} \mathrm{Ga}_{0.8} \mathrm{~N} / \mathrm{GaN}$ interface were investigated by magnetotransport measurements at low temperatures and magnetic fields up to $15 \mathrm{~T}$ in a ${ }^{3} \mathrm{He}$ refrigerator. The sample was immersed in liquid helium. The temperature could be cooled down to as low as $0.3 \mathrm{~K}$ and be controlled from 0.3 to $1.2 \mathrm{~K}$ by carefully adjusting the heater. It is also possible to reach higher temperatures up to $4.3 \mathrm{~K}$ by adjusting the ${ }^{4} \mathrm{He}$ absorption rate. The data were taken by conventional lock-in techniques. During the measurements, the current was monitored and kept small to avoid electron-heating effects (smaller than $1 \mu \mathrm{A})$.

SdH measurement has been a very welcomed tool for studying the properties of two-dimensional carriers, such as effective masses, carrier concentrations, and scattering times. Figure 1(a) shows typical Shubnikov-de Haas oscillations taken at $0.3 \mathrm{~K}$. There is only one series of oscillations and the $\mathrm{SdH}$ oscillations can be resolved from 6 to $15 \mathrm{~T}$.

The oscillatory part of the SdH oscillations can be expressed as ${ }^{1}$

$$
\Delta \rho_{X X} \approx 4 \rho_{0} \frac{Z}{\sinh (Z)} \exp \left(-\pi / \omega_{C} \tau\right)
$$

where $Z=2 \pi^{2} k_{B} T / \hbar \omega_{C}, \omega_{C}=e B / m^{*}$, and $\tau$ is the quantum lifetime. 


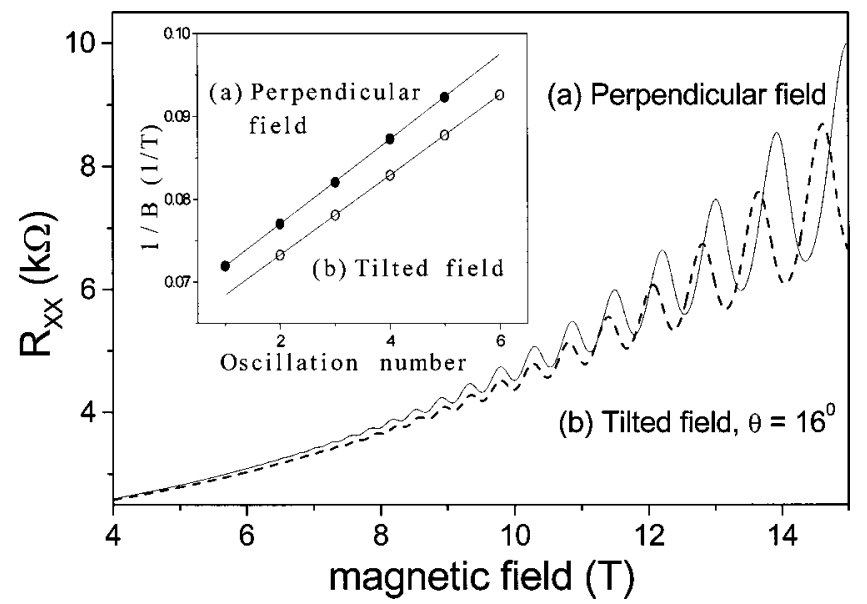

FIG. 1. Typical SdH oscillations at temperature $0.3 \mathrm{~K}$ : (a) perpendicular field (solid line) and (b) tilted field of $\theta=16^{\circ}$ (dotted line). The inset shows the inverse magnetic field of the $\mathrm{SdH}$ maximum as a function of the successive oscillation numbers: (a) result for perpendicular field (solid circles) and (b) result for tilted field (open circles).

The inverse magnetic fields of the $\mathrm{SdH}$ oscillation maxima as a function of the successive oscillation numbers are plotted as solid circles in the inset of Fig. 1. The data can be described by the simple linear equation ${ }^{3}$

$$
\frac{1}{B_{N}}=N \frac{e}{\pi \hbar n}+C
$$

where $N$ is an integer, $C$ is a constant, and $n$ is the carrier concentration. The choice of the oscillation number is arbitrary. The solid lines in the inset of Fig. 1 show the fitting to Eq. (2). The slope of the line corresponds to the carrier density $9.4 \times 10^{12} \mathrm{~cm}^{-2}$.

To verify the two-dimensionality of the conducting carriers, we rotated the sample orientation against the magnetic field. Figure 1(b) shows the $\mathrm{SdH}$ oscillations taken for $\Theta$ $=16^{\circ}$ inclined with respect to the magnetic field. The corresponding minimum positions of the $\mathrm{SdH}$ oscillations shift along with $B \cos \Theta$. The hollow circles in the inset of Fig. 1 show the inverse magnetic fields of the $\mathrm{SdH}$ maxima as a function of the successive oscillation numbers. The $2 \mathrm{D}$ character is further confirmed by examining the $\cos \Theta$ value of the slope ratio between the lines obtained from the tilted and perpendicular magnetic fields. It is in good agreement within the precision of our sample holder geometry.

Several selective temperature-dependent $\mathrm{SdH}$ oscillations are shown in Fig. 2. The oscillation amplitudes can be found to increase with the decrease of temperature. The effective mass can be extracted from the variation of the amplitude of the SdH oscillation with temperature. From Eq. (1),

$$
\frac{A_{1}\left(B, T_{1}\right)}{A_{2}\left(B, T_{2}\right)}=\frac{Z_{1} \sinh Z_{2}}{Z_{2} \sinh Z_{1}},
$$

where $A_{i}$ is the amplitude of the oscillation and $B$ is the magnetic-field value where the amplitudes are taken. The effective masses are obtained by solving Eq. (3). It is also possible to fit the amplitude by the conventional $\ln (A / T)$ vs $T$ plot. ${ }^{3}$ The fitting for the effective mass for two magneticfield values is shown in the inset of Fig. 2. The results of the calculated effective mass are shown in Fig. 3. It is found that

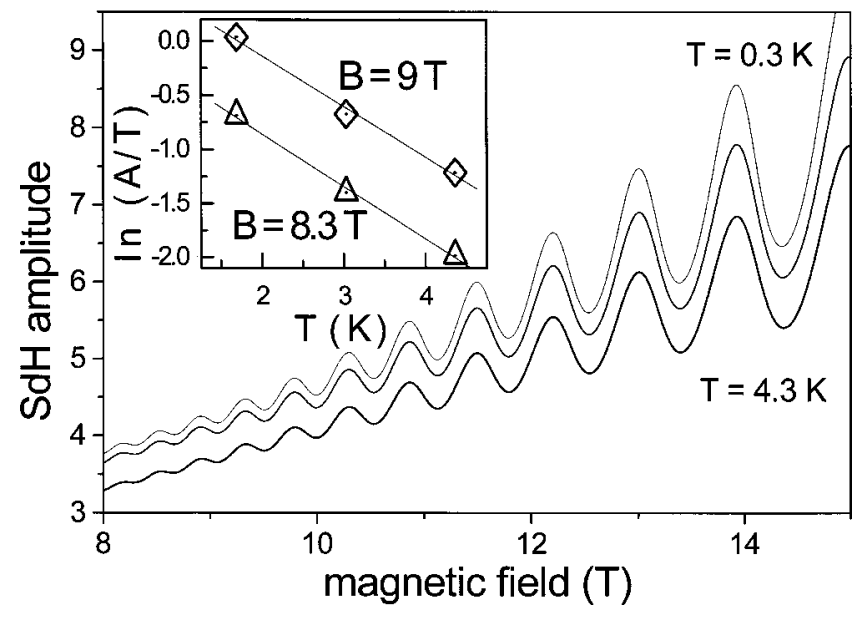

FIG. 2. Temperature-dependent SdH oscillations obtained at $0.3,1.3$, and $4.3 \mathrm{~K}$ (from top to bottom). The inset shows the fitting for the effective mass for two magnetic-field values, 8.3 and $9 \mathrm{~T}$, and yields the effective-mass values 0.246 and 0.253 , respectively.

the effective mass increases with the magnetic field. The enhancement of the effective mass can be described by a simple linear fit: $m^{*} / m_{0}=0.17+0.0092 B$, or a nonlinear fit which includes a quadratic term: $m * / m_{0}=0.195+0.042 B$ $+0.00025 B^{2}$, which is plotted as the dashed and the solid lines in Fig. 3, respectively. Both approaches give good fits with the data points. The mass variation is a consequence of the nonparabolicity of the conduction band. The increase of the magnetic field enlarges the electron wave vector $k_{\|}$in the plane perpendicular to the magnetic field. Then, higher terms of the wave vector are required to describe the physics when the magnetic field is large. The effective mass is, therefore, altered since the effective mass contains information on the band structure.

It was as early as the late 1950s that Kane ${ }^{8}$ and Wallis ${ }^{9}$ successively developed the theoretical approach to include $k^{4}$ terms in the conduction-electron energy expansion, which implied a variation of effective mass with magnetic field. It was experimentally observed by Palik and co-workers, ${ }^{10,11}$ who studied the effective mass in $n$-type InSb. Palik found that the effective mass increases roughly

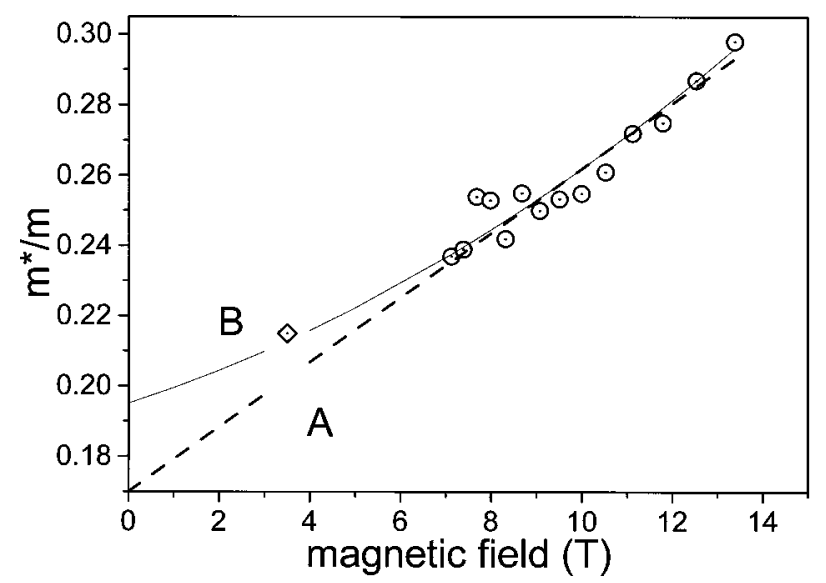

FIG. 3. Calculated effective mass at some selected fields of maximum $\mathrm{SdH}$ peaks (open circles). The effective mass increases with the magnetic field. Dash line A is a simple linear fit: $m^{*} / m_{0}=0.17+0.0092 B$, while solid line B is the nonlinear fit: $m * / m_{0}=0.195+0.0042 B+0.00025 B^{2}$. The low-field data reported by Saxler et al. (see Ref. 3) are shown as an open diamond. 
TABLE I. Measured sheet carrier concentrations and effective masses (in units of free-electron mass $m_{0}$ ) obtained from previous magnetotransport and cyclotron resonance (CR) experiments. The masses obtained by the two experimental methods give different results. The last two rows show the theoretical results of the first-principle calculation and tight-binding method. In our results, a linear extrapolation to zero field with Ando's correction yields an effective-mass value of $0.1825 \pm 0.0125$ of the free-electron mass.

\begin{tabular}{lccc}
\hline \hline \multicolumn{1}{c}{ Reports } & Approaches & $\begin{array}{c}\text { Carrier } \\
\text { concentration }\left(\mathrm{cm}^{-2}\right)\end{array}$ & $\begin{array}{c}\text { Effective mass } \\
\text { (without correction) }\end{array}$ \\
\hline Ref. 3 (Saxler) & $\mathrm{SdH}$ & $5.1 \times 10^{12}$ & 0.215 \\
Ref. 1 (Wong) & $\mathrm{SdH}$ & $5.4 \times 10^{12}$ & 0.228 \\
Ref. 4 (Brana) & $\mathrm{SdH}$ & $1.01 \times 10^{13}$ & 0.23 \\
Our results & $\mathrm{SdH}$ & $9.4 \times 10^{12}$ & 0.23 (low field) \\
Ref. 5 & $\mathrm{CR}$ & $3.7 \times 10^{12}$ & 0.242 \\
Ref. 2 & $\mathrm{CR}$ & $3.6 \times 10^{12}$ & 0.242 \\
Ref. 6 & First-principle result & & 0.18 \\
Ref. 7 & Tight-binding & & 0.18 \\
\multicolumn{4}{c}{ method } \\
\hline \hline
\end{tabular}

linearly with the magnetic field and he successfully interpret his results in terms of the Kane-Wallis (KW) approach together with consideration of band interactions since the $\mathrm{KW}$ approach solely yields a slightly parabolic behavior with the magnetic field when the magnetic field exceeds $3 \mathrm{~T}^{10}$ Similarly, behavior was observed for different material systems, Whall et al. ${ }^{12}$ studied remote doped $\mathrm{Si} / \mathrm{SiGe}$ and found that the effective-mass variation with the magnetic field is roughly linear for a magnetic field lower than $3 \mathrm{~T}$. Thus, we attribute the mass variation with the magnetic field mainly to the conduction-band nonparabolicity.

Because we do not have data taken in a lower field, it is difficult to determine which fit is better by our data alone. However, Saxler et al. ${ }^{3}$ reported an effective-mass measurement taken at magnetic fields between 3 and $4 \mathrm{~T}$. We, therefore, plot their data as an open diamond in Fig. 3. It may be seen that the data prefers the nonlinear fit. It suggests that the nonlinear fit would give a better description of the magneticfield-induced nonparabolicity. The mass of the extrapolation to zero field is $m^{*}=0.195 \pm 0.005 m_{0}$ for the nonlinear fit. The values are smaller than most experimental outcomes previously reported. Since a triangular potential well is formed by the accumulated electrons in the GaN layer close to the interface, a further correction can be made by taking Ando's formula into account to estimate its contribution to the mass enhancement. The effective mass is lowered by $0.01 m_{0}$ to $0.185 \pm 0.005 m_{0} \cdot{ }^{4}$ It is remarkably close to the results obtained by first-principles calculations given by Suzuki $^{6}$ and by the tight-binding method. ${ }^{7}$ In our measurements, the effective mass obtained at relatively low magnetic fields is about $0.23 m_{0}$, which is very close to the recent reported value. ${ }^{3,4}$ We, therefore, suggest that most previously reported experimental results contain an additional contribution due to magnetic field. When the magnetic-field component is removed from the data, the effective mass approaches the predictions made by first-principle calculations. This is clearly shown in Table I, where we summarize the reported effective mass by other groups and ours obtained from magnetotransport measurements. Thus, a complete understanding of the transport measurements of $\mathrm{AlGaN} / \mathrm{GaN}$ must incorporate the effect due to the magnetic field.
In conclusion, magnetotransport measurements have been performed on electrons confined in a Si-doped GaN/ AlGaN heterostructure. SdH oscillations were evidence of the $2 \mathrm{DEG}$ in the heterostructure and were further confirmed for their two-dimensional character. The sheet carrier concentration determined from $\mathrm{SdH}$ oscillations was 9.4 $\times 10^{12} \mathrm{~cm}^{-2}$. The effective mass was studied as a function of the magnetic field. The measured effective mass was found to increase with the magnetic field and was taken as evidence of conduction-band nonparabolicity. The extrapolation of the experimental data and the correction of Ando's formula together give $m^{*}=0.185 \pm 0.005 m_{0}$, which is in excellent agreement with the prediction made by first-principle calculations. Our results suggest that magnetic-field-induced band nonparabolicity must be taken into account in magnetotransport experiments and is responsible for the discrepancies between the theory and previous experimental results.

This work was partly supported by the National Science Council of the Republic of China.

${ }^{1}$ L. W. Wong, S. J. Cai, R. Li, K. Wang, H. W. Jiang, and M. Chen, Appl. Phys. Lett. 73, 1391 (1998)

${ }^{2}$ W. Knap, E. Frayssinet, M. L. Sadowski, C. Skierbiszewski, D. Maude, V. Falko, M. Asif Khan, and M. S. Shur, Appl. Phys. Lett. 75, 3156 (1999).

${ }^{3}$ A. Saxler, P. Debray, R. Perrin, S. Elhamri, W. C. Mitchel, C. R. Elsass, I. P. Smorchkova, B. Heying, E. Haus, P. Fini, J. P. Ibbetson, S. Keller, P. M. Petroff, S. P. DenBaars, U. K. Mishra, and J. S. Speck, J. Appl. Phys. 87, 369 (2000).

${ }^{4}$ A. F. Braña, C. Diaz-Paniagua, F. Batallan, J. A. Garrido, E. Muñoz, and F. Omnes, J. Appl. Phys. 88, 932 (2000).

${ }^{5}$ W. Knap, S. Contreras, H. Alause, C. Skierbiszewski, J. Camassel, M. Dyakonov, J. L. Robert, J. Yang, Q. Chen, M. Asif Khan, M. L. Sadowski, S. Huant, F. H. Yang, M. Goiran, J. Leotin, and M. S. Shur, Appl. Phys. Lett. 70, 2123 (1997)

${ }^{6}$ M. Suzuki, T. Uenoyama, and A. Yanase, Phys. Rev. B 52, 8132 (1995).

${ }^{7}$ T. Yang, S. Nakajima, and S. Sukai, Jpn. J. Appl. Phys., Part 1 34, $5912(1995)$

${ }^{8}$ E. O. Kane, J. Phys. Chem. Solids 1, 249 (1956).

${ }^{9}$ R. F. Wallis, J. Phys. Chem. Solids 4, 101 (1958).

${ }^{10}$ E. D. Palik, G. S. Picus, S. Teitler, and R. F. Wallis, Phys. Rev. 122, 475 (1961).

${ }^{11}$ E. D. Palik, S. Teitler, and R. F. Wallis, J. Appl. Phys. 32, 2132 (1961).

${ }^{12}$ T. E. Whall, A. D. Plews, N. L. Mattey, P. J. Phillips, and U. Ekenberg, Appl. Phys. Lett. 66, 2724 (1995). 